\title{
COMMENT LES RELATIONS POLICE-POPULATION PEUVENT-ELLES DEVENIR CONFLICTUELLES ? VERS UNE IDENTIFICATION DES FACTEURS DE RISQUE EN PROVINCE DE LIĖGE
}

\author{
Lisa Pelssers
}

Presses de Sciences Po | «Sociologies pratiques »

2021/2 N 42 | pages 117 à 119

ISSN 1295-9278

ISBN 9782724637090

Article disponible en ligne à l'adresse :

https://www.cairn.info/revue-sociologies-pratiques-2021-2-page-117.htm

Distribution électronique Cairn.info pour Presses de Sciences Po.

(C) Presses de Sciences Po. Tous droits réservés pour tous pays.

La reproduction ou représentation de cet article, notamment par photocopie, n'est autorisée que dans les limites des conditions générales d'utilisation du site ou, le cas échéant, des conditions générales de la licence souscrite par votre établissement. Toute autre reproduction ou représentation, en tout ou partie, sous quelque forme et de quelque manière que ce soit, est interdite sauf accord préalable et écrit de l'éditeur, en dehors des cas prévus par la législation en vigueur en France. Il est précisé que son stockage dans une base de données est également interdit. 


\title{
Comment les relations police-population peuvent-elles devenir conflictuelles? \\ Vers une identification des facteurs de risque en Province de Liège
}

\author{
Lisa Pelssers ${ }^{1}$
}

Depuis plus de vingt ans, les politiques de sécurité en Belgique se sont graduellement déplacées de la répression et de la lutte contre la délinquance vers une police de prévention et de proximité (Loubet del Bayle, 2001). Cette nouvelle perspective vise une plus grande souplesse des services de police dans leurs prestations et conduit à une diversification croissante de leurs interventions, parfois assez éloignées des fonctions policières initiales (Smeets et Tange, 2009). Le développement de relations et de collaborations entre la police et la société rencontre toutefois diverses sources de résistance. En particulier, cette politique de rapprochement n'empêche pas les forces de l'ordre d'être tantôt critiquées par l'opinion publique (Boivin et Lam, 2016), tantôt la cible d'actes de violence (Ocqueteau, 2008 ; Moreau de Bellaing, 2009).

Dès lors, comment les policiers perçoivent-ils et expliquent-ils l'émergence de la violence dans leur contexte de travail ? C'est cette question que traite le présent mémoire en analysant divers facteurs de risque qui, selon les policiers, engendrent des processus d'escalade conflictuelle avec la population. Tout d'abord, l'étude repose sur des entretiens informels avec différents acteurs de la police $(n=16)$ qui ont été menés entre août 2019 et juin 2020. Ils s'inscrivent dans une démarche exploratoire qualitative qui nous a permis d'élaborer un questionnaire puis d'éclairer les résultats quantitatifs obtenus. L'enquête par questionnaire $(n=166)$ a été menée entre mars et mai 2020 auprès d'un échantillon de 155 policiers de l'intervention provenant de 14 zones de police de la Province de Liège. Ensuite, deux techniques statistiques ont été employées pour conduire l'analyse. D'une part, le test du Chi-2 d'indépendance a permis de relever les facteurs conduisant au processus d'escalade conflictuelle. D'autre part, le modèle de régression logistique multinomiale a permis de mesurer l'influence de chacun de ces facteurs une fois combinés.

Ainsi, ce travail s'est uniquement concentré sur les policiers de terrain faisant de l'intervention. C'est également ce qui sera sous-entendu lorsqu'il sera fait mention de police ou de policiers. L'intervention constitue une des sept activités confiées à la police locale depuis la mise en place de la politique de proximité au début des années 2000. Les autres activités sont le travail de quartier, l'accueil, l'assistance policière aux victimes, la recherche et l'enquête locale, la gestion négociée de l'espace public (anciennement appelée "maintien de l'ordre ») et la circulation routière (Tange, 2000).

1. Maîtresse de conférences, Centre de recherche et d'interventions sociologiques (CRIS), Université de Liège. 
Un premier résultat de l'étude met en lumière le décalage constaté entre les discours tenus à propos de la police de proximité et les pratiques observées sur le terrain. Selon Jérémie Gauthier (2017), ce décalage constitue un facteur de risque d'escalade conflictuelle car les policiers disent ressentir une incohérence entre les discours politiques, les attentes de leur hiérarchie et les moyens mis à leur disposition pour effectuer concrètement leur travail quotidien. Ce sentiment de disjonction influence négativement leur relation avec la population.

Deuxièmement, les analyses indiquent que les policiers interrogés souffrent d'un manque de reconnaissance de la société en général. Ce constat n'est pas uniquement relevé dans leurs interactions avec les citoyens mais également dans celles avec des acteurs extérieurs tels que les journalistes, les travailleurs sociaux et même leur hiérarchie (Delpeuch et al., 2017). Cette dernière peut toutefois jouer un rôle important en répondant à ce besoin de reconnaissance, d'autant plus que la surcharge de travail constitue de manière significativee un facteur de risque, tant statistiquement (dans les régressions observées) que lors des échanges informels avec les policiers de l'intervention.

Troisièmement, les policiers questionnés considèrent le travail de prévention et de proximité comme "une fonction de service social plus qu'un vrai travail policier ". La plupart aspirent à un rôle de "chasseurs de crime " plutôt que d' "agent de la paix publique ". Ceci passe, notamment, par la multiplication des contrôles d'identité proactifs ou encore par la visualisation d'un groupe vu comme hostile au travers d'une manifestation. Ce dernier facteur a, par ailleurs, été relevé comme facilitant significativement le processus d'escalade conflictuelle.

Quatrièmement, les policiers d'intervention ne considèrent pas la gestion de conflits comme une aptitude qui peut s'apprendre. En effet, ils s'accordent à dire qu'« on ne peut pas former à la gestion de conflits, une grande partie est une question de caractère et l'autre partie s'apprend sur le terrain ". Ceci explique sans doute pourquoi, statistiquement parlant, autant de facteurs de risque relationnels et individuels sont ressortis comme influençant significativement le processus d'escalade conflictuelle. En effet, faire preuve d'empathie et de souplesse réduirait le risque de tels processus alors que l'utilisation d'exigences et de contraintes, la difficulté d'adopter le point de vue d'une autre personne, tout comme le stress et l'irritabilité sont autant de facteurs qui en augmenteraient le risque.

II est important de préciser que les facteurs identifiés s'enchevêtrent et doivent être pris en considération simultanément. Ce travail s'inscrit dès lors dans une approche pluraliste associant les différentes postures des policiers de l'intervention à l'égard de leurs différents publics à une variété de facteurs (Delpeuch et al., 2017). Dans notre recherche, tous ces facteurs, bien que non exhaustifs, contribuent à expliquer pourquoi des missions, en théorie semblables, sont traduites différemment en pratique. Ceci conduit alors, parfois, au développement d'escalades conflictuelles. Cette recherche et l'analyse des données recueillies rendent particulièrement bien compte de la diversité des pratiques policières et de l'adaptabilité dont les policiers doivent faire preuve. La culture policière se construit donc comme le produit de l'avenir qui leur est destiné dans cette institution dont ils sont les rouages, et des confrontations quotidiennes inhérentes à leur fonction, telles les contraintes qui émergent dans leur environnement de travail (le froid, les doubles nuits, la charge psychosociale, le stress, etc.), les expériences 
Comment les relations police-population peuvent-elles devenir...

vécues face aux enjeux et les contingences irréductibles de chaque situation ainsi que les appréhensions par rapport aux tâches risquées qu'ils doivent exécuter.

Cette étude rend ainsi compte de la coproduction sociopolitique des rapports entre police et citoyens, déterminés par des choix politiques opérés au sommet, matérialisés par la hiérarchie locale et, enfin, réappropriés par chaque policier selon son vécu et ses spécificités (Delpeuch et al., 2017). Et ce sont précisément ces multiples facettes du travail policier qui compliquent l'identification des facteurs pouvant influencer négativement leur interaction avec le public.

lisa.pelssers@uliege.be

\section{Références bibliographiques}

Ardoino J. (1965), Propos actuels sur l'éducation, Paris, L'Harmattan, coll. " Hommes et organisations".

BoIvin R., LAM, R. (2016), "Sondages Web, opinion publique et performance policière ", Criminologie, 49 (1), p. 225-245.

Bourton W., DI Prima C. (2018, mai 30), «Le policier, mal-aimé et glorifié », Le Soir Plus, https://plus.lesoir.be

DelPEUCH T., Ross J., BonNet F. (2017), « Les analyses sociologiques des relations police/population. Vers une reconnaissance de la variété des pratiques policières », Droit et société, 97 (3), p. 457-68, doi :10.3917/drs.097.0457

GAUTHIER J. (2017), « Le policier et le culturel. Police et minorités à Berlin », Cultures et Conflits, 107, p. 123-141.

LOUBET DEL BAYLE J.-L. (2001), «Vers une monopolisation policière du contrôle social », Cahiers de la sécurité intérieure, 44, p. 207-225.

Moreau de Bellaing C. (2009), "Comment la violence vient aux policiers ", Genèses, 2, p. 24-44.

OCQUeTEAU F. (2008), Violences en actes dans les rapports entre mineurs et policiers, Archives de politique criminelle, 1, p. 149-162.

Smeets S., Tange C. (2009), Le Community Policing en Belgique. Péripéties de la constitution d'un modèle de travail policier, Genève, Geneva Centre for the Democratic Control of Armed Forces (DCAF).

TANGE C. (2000), "La police de proximité », Courrier hebdomadaire du CRISP, 1691-1692, p. 1-63, https://doi.org/10.3917/cris.1691.0001 